AGGRESSIVE BEHAVIOR

Volume 23, pages 1-10 (1997)

\title{
Effects of Provocation on Emotions and Aggression in Males
}

\author{
Stephen T. Chermack, ${ }^{1 *}$ Mitchell Berman, ${ }^{2}$ and Stuart P. Taylor ${ }^{3}$ \\ ${ }^{1}$ Alcohol Research Center, Department of Psychiatry, University of Michigan, Ann Arbor, Michigan \\ ${ }^{2}$ Medical College of Pennsylvania and Hahnemann University, Philadelphia, Pennsylvania \\ ${ }^{3}$ Psychology Department, Kent State University, Kent, Ohio
}

: : : : : : : : : : : : : : : : : : : : : : : : : : : : : : : :

The effects of provocation of emotions and human physical aggression were examined using a competitive reaction time paradigm. Fifty-four males participated in the competitive task with a fictitious opponent. Participants were assigned to two conditions, low-constant provocation and increasing provocation. Aggression was defined as the intensity of electric shocks participants selected for the opponent to receive. Negative emotions (threat, harm) and positive emotions (benefit, challenge) were assessed at four times during the experimental task. The results revealed that both aggressive responding and harm emotions differed as a function of opponent's level of provocation. There was little compelling evidence that emotions served as an important intervening variable in the relationship between provocation and aggression. Aggr. Behav. 23:1-10, 1997. ๑) 1997 Wiley-Liss, Inc.

: : : : : : : : : : : : : : : : : : : : : : : : : : : : : : :

Key words: aggression; affect; emotions; provocation

\section{INTRODUCTION}

There is considerable controversy about the contributions of social perceptions and emotional states in the elicitation of aggression. Cognitively oriented theorists emphasize perceptions of the social environment as direct antecedents of aggression [Ferguson and Rule, 1983]. Laboratory studies have demonstrated that the belief that another person's actions are intended to be provocative can result in both anger [Betancourt and Blair, 1992] and aggression [Greenwell and Dengerink, 1973; Ohbuchi and Kambara, 1985; Epstein and Taylor, 1967]. Emotion-oriented theorists suggest that emotional states are the primary determinant of aggressive behavior. For example, Berkowitz [1989] argued that "any kind of negative affect, sadness as well as depression and agitated irritability, will produce aggressive inclination ..." Researchers have also demonstrated that positive emotions can reduce aggressive tendencies [Sapolsky, 1984; Zillman and Bryant, 1984].

Received for publication 24 January 1996; accepted 25 March 1996.

*Correspondence to: Stephen Chermack, the University of Michigan Alcohol Research Center, Department of Psychiatry, 400 East Eisenhower Parkway, Suite A, Ann Arbor, MI 48108.

(C) 1997 Wiley-Liss, Inc. 
Emotional states have been proposed to be important intervening variables in the relationship between provocation and aggression [Berkowitz, 1993]. For example, Buss and Perry [1992] argued that anger may be the link between the cognitive component of aggression (hostility) and the instrumental component (overt aggression). A recent study by Betancourt and Blair [1992] demonstrated that both cognitive-attributional factors (perceived intentionality and controllability of instigative behaviors) and emotions were related to a self-report measure of aggressive intentions. However, this study did not include a behavioral measure of overt aggression. Therefore, it could not be determined whether emotions were related to physical aggression.

Research on the effects of provocation has generally focused on monitoring either behavioral aggression or emotional reactions [e.g., Betancourt and Blair, 1992; Hammock and Richardson, 1992]. Studies that have examined both have measured emotional reactions to provocation either before or after the completion of an aggressive encounter [e.g., Epstein and Taylor, 1967; O’Leary and Dengerink, 1973; Zeichner et al., 1994]. No study has examined the effects of provocation on both a behavioral measure of aggression and concomitant emotional states during an aggressive altercation. Furthermore, few studies have attempted to distinguish the effects of provocation from other variables that could presumably result in the escalation of aggression. For example, escalation of overt aggression has been observed even without increases in provocation [e.g., Goldstein et al., 1975]. Goldstein et al. [1975] speculated that increases in aggression over time may be due to a "disinhibition of aggressive impulses." In contrast, several studies have demonstrated that the escalation of aggression is a function of increased provocation, rather than the passage of time [Taylor, 1967; Epstein and Taylor, 1967]. Thus, there is some controversy about whether changes in emotions and expressed aggression during aggressive encounters are a function of provocation or disinhibition associated with time spent in an aggressive encounter.

The purpose of this study was to investigate the effects of provocation on both aggressive behavior and self-reported emotional reactions at various points during an aggressive encounter. The aggressive encounter occurred in the context of a competitive reaction-time (RT) task [see Taylor, 1967]. The competitive RT task provides a context in which the aggressive intent of both the participant and the opponent is clearly communicated by delivery of various levels of electric shock.

The RT task used in this study appears to provide a valid measure of physical aggression. This paradigm has been demonstrated to discriminate between groups of participants theoretically expected to differ in aggression, e.g., psychopathic individuals [Dengerink, 1971], prejudiced persons [Genthner and Taylor, 1973], and participants with aggressive tendencies [Hammock and Richardson, 1992], and to be sensitive to situational factors that are expected to affect aggressive responding, such as social pressure [Borden and Taylor, 1973] and provocation [Hammock and Richardson, 1992]. Furthermore, studies using the competitive RT paradigm have demonstrated the facilitation of aggression by ethanol [see Taylor and Chermack, 1993, for a review].

Participants competed in the RT task with either an increasingly provocative (high aggressive intent) or consistently low-provocative (low aggressive intent) fictitious opponent. Aggression was defined as the magnitude of shock participants were willing to administer to their adversary. To address limitations of previous studies in which emotions have been measured either before or after an aggressive encounter, both negative and positive emotions were assessed several times during the encounter. Emotions 
were measured with a questionnaire developed by Folkman and Lazarus [1985]. This questionnaire includes two scales measuring negative emotions (harm and threat), and two scales measuring positive emotions (challenge and benefit). In addition to assessing the effects of provocation on aggression and emotions, the relationship between the negative and positive emotional states and subsequent aggressive behavior during the competitive task was examined. It was expected that increasing provocation would result in higher levels of both aggression and negative emotions, and reduced levels of positive emotions. In addition, it was expected that individual differences in emotional states during the task would be related to the level of aggressive responding.

\section{MATERIALS AND METHODS Participants}

Participants were 54 male undergraduates enrolled in an introductory psychology course. They earned extra credit points in their psychology class for being in the study. They were informed that the experiment investigated the effects of competition on perception and reaction time. The methods and procedures utilized in this study were approved by the University's Human Subject Review Board.

\section{Procedure}

Upon arrival to the experimental setting, participants were escorted to a cubicle containing a chair and a desk, and informed consent was obtained. They were assigned to either a low constant provocation (LCP) or increasing provocation (IP) condition. The participants next were escorted to the experimental room and seated before the RT task apparatus. A concentric ring shock electrode was attached to their nondominant wrist.

Following a 5-min delay during which the experimenter presumably was preparing the opponent for the task, the shock "unpleasantness" thresholds for the participant and the "opponent" were determined. Participants were then informed that they were competing in a RT task with another person in an adjoining room. They were told that at the beginning of each trial, they would select (by pressing one of ten buttons) the intensity of shock they wished their opponent to receive. The shock setting buttons were labeled 1 to 10 . The button labeled 10 corresponded to the participant's unpleasantness threshold. Number 9 was set at $95 \%$ of the maximum, number 8 at $90 \%$ of the maximum, 7 at $85 \%$ of the maximum, and so forth. Participants were informed that the shock would be administered to their opponent at the end of the RT trial if they were faster than their opponent, and that they would receive the shock their opponent had set for them if the opponent was faster. Thus, participants were informed that either they or their opponent would receive a shock, depending on the outcome of the competition, and that both could select the intensity of shock the other would receive. They were also informed that regardless of the outcome, at the end of each trial a feedback light indicated the shock intensity that had been set for them by their opponent. That way, participants could see the shock level their opponent had selected for them even if they did not receive the shock. In actuality, the opponent was simulated. The frequency of wins and losses (50\%) and the intensities of shock received were predetermined.

Participants competed in 21 RT trials, which were divided into three blocks. The three blocks consisted of Trials 2 through 7, 9 through 14, and 16 through 21. The initial trial shock setting was made in the absence of any provocation and was analyzed sepa- 
rately. Trials 8 and 15 were transition trials between blocks and were not included in the data analysis. The purpose of the transition trials was to enhance the credibility of the task by smoothing the transition between blocks. The degree of provocation experienced by the participants was determined by the level of shock their opponent set for them to receive during the competitive RT task. Participants in the LCP condition interacted with "opponents" who consistently set low shocks during all three blocks (average shock setting equaled 2.5). Participants in the IP condition interacted with "opponents" who set increasingly higher levels of shock. The average shock set by the opponent for blocks one to three were 2.5, 5.5, and 8.5, respectively. Each participant lost (received shock and feedback) on half of the trials in each block and one of the two transition trials.

Positive and negative emotions were assessed at four times during the course of the aggressive encounter, baseline (immediately prior to the first block of trials), and following each of the three blocks of RT trials. While participant's emotions were being assessed after each block of trials, the opponent's last shock setting remained illuminated. For LCP participants, this shock level was " 2 " after each block of trials. For the IP participants, the shock levels were 4, 7, and 9, respectively. Participants were asked to indicate on a 5-point Likert scale the extent to which they felt each of 15 emotions. The items were grouped into the following four emotions scales: benefit (exhilarated, pleased, happy, and relieved); challenge (confident, hopeful, and eager); harm (angry, sad, disappointed, guilty, and disgusted); and threat (worried, fearful, anxious). Participants' score for each scale consisted of the sum of their responses to the scale items. The mean coefficient alphas for these scales as reported by Folkman and Lazarus [1985] were .78 for the benefit scale, .59 for the challenge scale, .84 for the harm scale, and .80 for the threat scale. This questionnaire has been demonstrated to be sensitive to changes in emotions during the course of a stressful encounter in a sample of college students [Folkman and Lazarus, 1985]. Furthermore, Folkman and Lazarus [1985] found that challenge and threat emotions are associated with anticipations regarding stressful encounters, whereas harm and benefit emotions are associated with outcomes of such encounters. The benefit and challenge scales are considered measures of positive emotional states, whereas the threat and harm scales are considered measures of negative emotional states.

At the conclusion of the RT task, participants were also asked to fill out a questionnaire regarding their perceptions of the RT task. This questionnaire was designed to assess the following: the painfulness of shocks received, the importance of winning during the task, and qualitative questions designed to confirm that participants understood the task. After completion of the questionnaire, participants were debriefed and dismissed.

\section{RESULTS}

All analyses were conducted with two-tailed significance tests at the .05 level. Post hoc tests were conducted using Tukey's HSD test at the .05 level. A one-way analysis of variance (ANOVA) of initial trial shock setting was conducted to assess whether the IP and LCP groups differed in their shock settings prior to receiving feedback about their opponent's aggressive intentions. According to the analysis, the experimental groups did not differ significantly in their initial trial shock settings $(F<1)$. 
To determine the effects of provocation on shock setting, a 2 (provocation condition) $\times 3$ (blocks) repeated measures ANOVA was performed on the mean shock intensities set by the participants. According to this analysis, the main effect of provocation condition was significant, $F(1,52)=7.29, P<.01$. The mean (SD) shock settings for the LCP and IP groups were 3.99 (2.02) and 5.17 (1.05), respectively. The main effect of blocks was significant, $F(2,104)=63.10, P<.001$. The mean $(\mathrm{SD})$ shock settings for blocks 1 through 3 were 3.61 (1.53), 4.26 (1.81), and 5.61 (2.55), respectively. A significant provocation condition by blocks interaction was found, $F(2,104)=50.43, P<.001$. The interaction between provocation condition and blocks is displayed in Figure 1. Post hoc analyses revealed that IP participants set significantly higher levels of shock in each successive block, and that LCP participants showed no significant changes in shock setting from block 1 to block 3 . In addition, the post hoc analyses revealed that the shock setting of LCP and IP participants differed significantly in only the third block of RT trials. The results of these analyses indicate that the aggressive intent of the opponent accounted for participants' aggressive behavior, rather than time spent in the encounter or competitive nature of the task.

A series of 2 (provocation condition) $\times 4$ (time) repeated measures ANOVAs were conducted to examine the effects of provocation on the four emotion scales. For the harm emotions scale, only the interaction of provocation condition and time was significant, $F(3,156)=4.80, P<.05$. The means (SD) for Time 1 through 4 harm emotions for the LCP group were 3.47 (4.58), 3.79 (3.36), 3.29 (3.15), and 2.68 (2.74), respectively. For the IP group, the means (SD) for Time 1 through 4 were 4.17 (4.16), 4.68 (3.08), 4.77 (3.16), and 6.06 (3.78), respectively. Post hoc tests indicated that the IP and LCP participants differed in harm emotions only at Time 4 (after the third block of RT trials), in which the IP participants had higher ratings than the LCP participants. For the

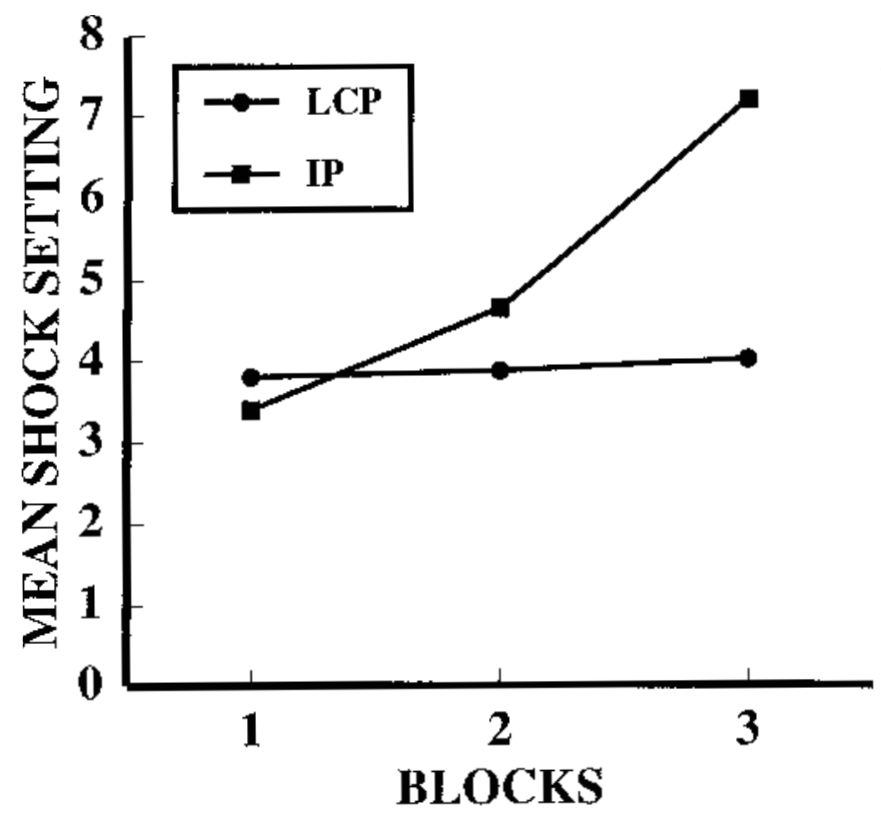

Fig. 1. Mean shock setting as a function of provocation condition and blocks. 
benefit emotions scale, the main effect of time was significant, $F(3,156)=6.18, P<.01$. The means for Time 1 through 4 were 5.98 (3.85), 7.81 (3.46), 6.93 (3.31) and 7.20 (3.51), respectively. According to post hoc tests, benefit emotions increased significantly between Time 1 and 2 . The interaction of provocation condition and time was also significant, $F(3,156)=2.70, P<.05$. The means (SD) for Time 1 through 4 benefit emotions for the LCP group were 5.96 (3.61), 8.37 (3.71), 8.07 (3.07), and 7.89 (3.38), respectively. For the IP group, the means (SD) for Time 1 through 4 were 6.00 (4.14), 7.26 (3.16), 5.78 (3.19), and 6.52 (3.58), respectively. Post hoc analyses revealed that the IP group had significantly lower ratings of benefit emotions than the LCP group only at Time 3 (following the second block of RT trials). There were no significant main effects or interactions for the perceived threat and challenge scales.

After the RT task, participants were asked to fill out a questionnaire regarding their perceptions of the task. One question asked participants to rate how important it was for them to win during the task on an 8-point Likert scale ranging from 1 (not at all) to 8 (very important). An ANOVA indicated that the participants in the IP group had significantly higher ratings $(\mathrm{M}=6.52, \mathrm{SD}=1.53)$ of the importance of winning than participants in the LCP group $(\mathrm{M}=5.44, \mathrm{SD}=2.28), F(1,52)=4.14, P<.05$. This raises the possibility that participants who were increasingly provoked may have been more aggressive due to a greater motivation to win. To further examine whether motivation to win was related to aggression, bivariate correlations between this item (importance of winning) and shock setting were conducted separately for both the LCP and IP groups. For the LCP group, the importance of winning was significantly correlated with block 2 and block 3 shock setting at the .05 level, $r=.39$ and $r=.41$, respectively. This suggests that motivation to win may have been associated with aggression for participants interacting with a relatively nonprovocative opponent. However, for the IP group, the importance of winning was not correlated with block 1, block 2, or block 3 shock setting. Thus, although participants in the IP group had higher ratings of the importance of winning than their LCP counterparts, importance of winning was not associated with their shock-setting behavior.

Another question asked the participants to rate the painfulness of the shocks they received during the task on an 8-point scale, ranging from 1 (not at all) to 8 (very painful). An analysis of these ratings revealed that compared to LCP participants, IP participants rated the shocks as being significantly more painful, $F(1,52)=6.10, P<$ .05. The means for the LCP and IP participants were $3.00(\mathrm{SD}=1.00)$ and $3.74(\mathrm{SD}=$ $1.20)$, respectively. This raises the possibility that differences in aggression for the IP and LCP groups could be due to different levels of pain experienced. To further examine whether shock painfulness was related to aggression, bivariate correlations between this item (painfulness of shocks) and shock setting were conducted separately for both the LCP and IP groups. For each group, painfulness of the shocks was not significantly correlated with block 1, block 2, or block 3 aggression during the task. Thus, although the LCP and IP groups differed in ratings of shock painfulness, these ratings were not associated with their shock-setting behavior.

\section{Associations Between Emotions and Shock Setting}

Bivariate correlations between emotions and subsequent shock setting were performed to determine if individual differences in emotional states were associated with aggressive responding. Significant correlations would provide some evidence that emotional 
states were associated with subsequent aggressive responding. Furthermore, according to criteria outlined by Baron and Kenny [1986], significant correlations between emotions and subsequent aggressive behavior would be necessary, but not sufficient, to demonstrate that emotions mediate (account for) the relationship between provocation and aggression. However, none of the emotions scales were significantly correlated with subsequent blocks of shock selections. The highest correlation was between harm emotions measured prior to the third block of trials and block 3 shock settings $(r=.26)$, but this was not significant at the .05 level.

It is possible that emotions moderated the relationship between provocation and aggression. A significant interaction effect of provocation condition and emotions, after controlling for the main effects of these variables, would be sufficient to demonstrate moderation [Baron and Kenny, 1986]. In order to investigate whether any of the emotions moderated the relationship between provocation and aggression, regression analyses were performed according to procedures delineated by Baron and Kenny [1986]. A total of eight regression analyses were conducted to test for moderation of each of the four emotion scales (measured at Time 2 and Time 3) on subsequent aggression (block 2 and 3 shock setting, respectively). According to these analyses, there were no significant interactions between emotions and provocation condition at the .05 level of significance.

\section{DISCUSSION}

The purpose of this study was to examine the effects of provocation on aggression and emotional states during an aggressive encounter, and to examine the relationship between emotional states and subsequent aggression. The results demonstrated that provocation had a significant impact on participants' aggressive behavior and subjective emotions. Participants competing with an increasingly provocative opponent set significantly higher shocks as provocation increased, and were more aggressive than participants competing with an opponent exhibiting low levels of shock. The aggressive responding of participants in the LCP group was highly stable, and did not change significantly throughout the task. Regarding negative emotions, participants facing the increasingly provocative adversary had higher ratings of harm emotions after receiving substantial increases in provocation. However, perceived threat emotions did not appear to be affected by the degree of the opponent's provocation. For the positive emotions, benefit emotions increased between pretask baseline and following the first block of trials in which they received relatively mild shocks from the opponent. However, participants in the IP group had significantly lower ratings of benefit emotions than LCP participants after the opponent first displayed a marked increase in provocation. Challenge emotions did not appear to be affected by provocation, suggesting that participants in the two groups viewed the task as equally challenging. None of the positive or negative emotions directly influenced aggressive responding, or intervened in the relationship between provocation and aggression. Thus, the findings are inconsistent with theories which emphasize that negative and positive emotions play a primary role in the relationship between provocation and aggression [Berkowitz, 1989; Sapolsky, 1984].

It is notable that motivation to win appeared to be related to aggression for LCP participants, but not for IP participants. This suggests that motivation to win may have more of an influence on aggression under conditions of relatively low provocation. 
However, when provocation escalates to moderate and high levels, aggression appears to be a reaction to the opponent's escalating aggressive intentions rather than the participant's motivation to win. The results also indicated that the painfulness of shocks received was not associated with aggression for either group. This suggests that the effects of provocation on aggression in this study were not attributable to the LCP and IP participants' differences in motivation to win or the painfulness of shocks received.

The overall pattern of results appears to be consistent with current cognitively oriented perspectives regarding the effects of provocation on negative emotions and aggression [Ferguson and Rule, 1983; Taylor, 1986; Betancourt and Blair, 1992]. Specifically, as the opponents' intentions to harm escalated, as reflected in increases in their shock setting, participants became more aggressive and experienced increased negative emotions as measured by the harm scale. Lazarus [1991] suggested that in addition to the provocateur's intentions to harm, such negative emotions are also related to an individuals' involvement in an encounter. For example, participants would be more likely to experience negative emotions if they feel there is a goal at stake in an encounter. The present study offers some evidence that is consistent with this perspective. Relative to the LCP participants, the IP participants felt that it was more important for them to win during the RT task, and they rated the shocks as being more painful. Thus, it could be argued that the IP participants had greater investment in the outcome of the trials due to either a greater desire to win or to avoid unpleasant shocks, or both, and that this also influenced their ratings of harm emotions. It is also possible, however, that the higher ratings on these variables of IP participants were due to an after-the-fact attempt to justify their aggressive responding. This issue could be addressed in future studies by having participants rate their desire to win and the painfulness of the shocks received during the course of the RT task.

The finding that threat emotions were unrelated to increasing provocation was unexpected. However, the lack of a relationship between threat and provocation may reflect that threat emotions are anticipatory evaluations of a potentially harmful situation, as has been suggested by Folkman and Lazarus [1985], rather than evaluations of events that have already occurred. Thus, it is possible that participants in both the IP and LCP groups felt equally threatened since the opponent in both conditions had equal capacity to administer painful shocks. The finding that harm emotions increased with provocation is also consistent with Folkman and Lazarus' perspective, in that harm emotions are thought to be associated with events that have occurred.

There was only modest evidence that provocation was related to changes in positive emotions. For example, the IP participants had lower ratings of benefit emotions than LCP participants only at one time point, and challenge emotions did not vary as a function of provocation. Thus, the results demonstrated that provocation increases negative emotions associated with being harmed during an aggressive encounter, and may have a much more modest effect in reducing positive emotions during an aggressive encounter.

The lack of a relationship between emotional states and subsequent aggression in this study was unexpected. The results of this study suggest that both emotional and behavioral reactions are affected by provocation, but that emotions may not be the primary determinant of aggression. This implies that factors other than emotions, such as perceived intentionality and controllability of incitive acts, norms of reciprocity or motivational states (intention to harm), may play a more central role in accounting for the relationship between provocation and aggression. However, some caution is war- 
ranted in drawing such conclusions from this study. It is possible that more intense forms of provocation than used in this study, leading to more intense emotional reactions, would be necessary to demonstrate that emotions mediate the relationship between provocation and aggression. It is also possible that other measures of emotional states would reveal such a relationship. Lastly, although the sample size in this study was adequate to detect the effects of provocation on aggression and emotions, it is possible that larger sample sizes would reveal a significant influence of emotions on aggression. Thus, future research is needed to assess whether a similar pattern of results would be found with more intense levels of provocation or other measures of emotional states, to investigate other variables as potential mediators of the provocation-aggression relationship, and to further delineate the relative influence of cognitive, motivational, situational, and emotional factors on behavioral aggression.

\section{REFERENCES}

Baron RA(1971): Magnitude of victim's pain cues and level of prior anger arousal as determinants of adult aggressive behavior. Journal of Personality and Social Psychology 17:236-243.

Baron RM, Kenny DA (1986): The moderatormediator variable distinction in social psychological research: Conceptual, strategic, and statistical considerations. Journal of Personality and Social Psychology 51:1173-1182.

Berkowitz L (1989): Frustration-aggression hypothesis: Examination and reformulation. Psychological Bulletin 106:59-73.

Berkowitz L (1993): "Aggression: Its Causes, Consequences, and Control." New York: McGraw-Hill.

Betancourt H, Blair I (1992): A cognition (attribution)-emotion model of violence in conflict situations. Personality and Social Psychology Bulletin 18:343-350.

Borden R, Taylor S (1973): The social instigation and control of aggression. Journal of Applied Social Psychology 3:354-361.

Buss AH, Perry M (1992): The aggression questionnaire. Journal of Personality and Social Psychology 63:452-459.

Dengerink H (1971): Anxiety, aggression and physiological arousal. Journal of Experimental Research in Personality 5:223-232.

Epstein S, Taylor SP (1967): Instigation to aggression as a function of degree of defeat and perceived aggressive intent of the opponent. Journal of Personality 35:265-289.

Ferguson T, Rule B (1983): An attributional perspective on anger and aggression. In Geen RJ, Donnerstein EI (eds): “Aggression: Theoretical and Empirical Reviews.” New York: Academic Press. pp. 41-74.

Folkman S, Lazarus RS (1985): If it changes it must be a process: Study of emotion and cop- ing during three stages of a college examination. Journal of Personality and Social Psychology 48:150-170.

Genthner R, Taylor S (1973): Physical aggression as a function of racial prejudice and the race of the target. Journal of Personality and Social Psychology 27:207-210.

Goldstein JH, Davis RW, Herman D (1975): Escalation of aggression: Experimental studies. Journal of Personality and Social Psychology 31:162-170.

Greenwell J, Dengerink H (1973): The role of perceived versus actual attack in human physical aggression. Journal of Personality and Social Psychology 26:66-71.

Hammock GS, Richardson DR (1992): Predictors of aggressive behavior. Aggressive Behavior 18:219-229.

Lazarus RS (1991): "Emotion and Adaptation.” New York: Oxford University Press.

Ohbuchi K, Kambara T (1985): Attacker's intent and awareness of outcome, impression management, and retaliation. Journal of Experimental Social Psychology 21:321-330.

O'Leary M, Dengerink H (1973): Aggression as a function of the intensity and pattern of attack. Journal of Research in Personality 7:61-70.

Sapolsky BS (1984): Arousal, affect, and the aggression moderating effect of erotica. In Malamuth NM, Donnerstein EI (eds): "Pornography and Sexual Aggression.” Orlando, FL: Academic Press. pp. 85-113.

Taylor SP (1967): Aggressive behavior and physiological arousal as a function of provocation and the tendency to inhibit aggression. Journal of Personality 35:297-310.

Taylor SP (1986): The regulation of aggressive behavior. In Blanchard B, Blanchard C (eds): "Advances in the Study of Aggression," Vol 2. New York: Academic Press. pp.91-119.

Taylor SP, Chermack ST (1993): Alcohol, drugs and human physical aggression. Journal of Studies on Alcohol, Supplement No. 11:78-88. 
Zeichner A, Allen JD, Giancola PR, Lating M (1994): Alcohol and aggression: Effects of personal threat on human aggression and affective arousal. Alcoholism: Clinical and Experimental Research 18:657-663.

Zillman D, Bryant J (1984): Effects of massive exposure to pornography. In Malamuth NM, Donnerstein EI (eds): "Pornography and Sexual Aggression." Orlando, FL: Academic Press. pp. 115-138. 